\title{
Resistência de Genótipos de Sorgo a Contarinia sorghicola (Coq.) (Diptera: Cecidomyiidae) e Diatraea saccharalis (Fabr.) (Lepidoptera: Pyralidae) e Influência Sobre Parasitóides
}

\author{
Fernando M. Lara ${ }^{1}$, Mauro R. D. A. Foss ${ }^{1}$, Arlindo L. Boiça Jr. ${ }^{1}$ e José G. Trigo ${ }^{1}$ \\ ${ }^{1}$ Departamento de Entomologia e Nematologia, FCAV/UNESP, Rodovia \\ Carlos Tonanni, km 5, 14870-000, Jaboticabal, SP.
}

An. Soc. Entomol. Brasil 26(2): 327-333 (1997)

Resistance of Sorghum Genotypes to the Sorghum Midge Contarinia sorghicola (Coq.) and Sugarcane Borer Diatraea saccharalis (Fabr.) and Influence on Parasitoids

\begin{abstract}
The resistance of some sorghum genotypes (Sorghum bicolor) to Contarinia sorghicola (Coq.) and Diatraea saccharalis (Fabr.) was evaluated in the field, as well the influence of genotypes over the parasitism by Tetrastichus sp. (Hymenoptera: Eulophidae) and Cotesia flavipes (Cam.) (Hymenoptera: Braconidae). Two experiments were conducted, in different planting dates. AF-28 genotype was the most resistant to both pests, while TX-2567 was the most susceptible. Sorghum midge parasitism was higher $(36.7 \%)$ on BR-501 and AF-28 (20.0\%) than on the other genotypes. Parasitism of the sugarcane borer by C. flavipes was higher on BR-601 and 3DX-57 (50 and $40 \%$ )and was not observed on SC-3541 and TX-2567 genotypes.
\end{abstract}

KEY WORDS: Insecta, Cotesiaflavipes, Tetrastichus sp., host plant resistance.

RESUMO - Avaliou-se a resistência de genótipos de sorgo (Sorghum bicolor) a Contarinia sorghicola (Coq.) e Diatraea saccharalis (Fabr.) e sua influência sobre parasitóides. Foram realizados ensaios em duas épocas de plantio, anotando-se o número de insetos (pragas e parasitóides) e calculando-se a percentagem de parasitismo. O genótipo AF-28 destacou-se como o mais resistente às duas espécies pragas, ocorrendo o inverso com o TX-2567; as maiores percentagens de parasitismo de C. sorghicola por Tetrastichus sp. (Hymenoptera: Eulophidae) ocorreram em BR-501 (36,7\%) e AF-28 (20,0\%); as maiores percentagens de parasitismo de D. saccharalis por Cotesia flavipes (Cam.) (Hymenoptera: Braconidae) foram observadas em BR-601 e 3DX-57 $(50$ e $40 \%)$.

PALAVRAS-CHAVE: Insecta, Cotesia flavipes, Tetrastichus sp., resistência de plantas.

A produção de grãos, forragens e álcool fazem do sorgo, Sorghum bicolor, uma cultura de grande potencialidade. Entretanto, alguns problemas são observados, destacando dentre estes as pragas, como a mosca Contarinia sorghicola (Coq.) (Rossetto et al. 
1967; Lara et al. 1977a) e a broca Diatraea saccharalis (Fabr.) (Lara et al. 1980). Dentre os vários métodos de controle dessas pragas, encontram-se a utilização de variedades resistentes e o controle biológico, sendo que geralmente a integração desses dois métodos é vantajosa, com efeito positivo da planta sobre os inimigos naturais (Lara 1991).

Com respeito a $C$. sorghicola, a variedade AF-28 vem sendo estudada e utilizada, no Brasil e no exterior, desde que Rossetto \& Banzatto (1967) constataram sua resistência a essa praga; Lara et al. (1977a) observaram que essa resistência se manteve em diversas épocas de plantio, e notaram que, na média dessas épocas, o nível de parasitismo por Eupelmus popa Girault e por Tetrastichus spp. foi baixo. Campos et al. (não publicado) verificaram que nos genótipos Sart (suscetível) e AF-28 (resistente) ocorreram as maiores percentagens de parasitismo de $C$. sorghicola por Aprostocetus diplosidis Crawford, 1907. Moura et al. (não publicado), verificando a preferência desse parasitóide por partes da planta de sorgo, em olfatômetro, constataram que ele foi mais atraído por flores não fecundadas e grãos. Em ensaio semelhante, Lara et al. (1994) observaram que as flores fecundadas da variedade IPA-201 foram mais atrativas em relação a outras partes e fases da planta e que as panículas de AF-28 foram as que mais atraíram aquele parasitóide entre as variedades testadas.

Lara \& Boiça Jr. (1993) constataram a resistência de vários genótipos à mosca do sorgo, entre os quais IS-7386, IS-10606, IS521, TX-399-8, e IS-7152; como os mais suscetíveis encontraram IS-5873, IS-7312, IS7204 e IS-9586. Com relação a $D$. saccharalis, Lara et al. (1977b) constataram a resistência dos genótipos EA-177 e EA-95 entre 86 materiais avaliados; Lara et al. (1979) confirmaram a resistência do EA-177 e também da variedade AF-28; a resistência da AF-28 foi observada por inúmeros autores, entre eles Boiça Jr. \& Lara (1983) e Lara \& Perussi (1984).

Waquil et al. (não publicado) verificaram que as variedades Brandes e Dale portaram- se como resistentes à broca; a variedade Brandes também foi reportada como resistente por Boiça Jr. \& Lara (1983); outros genótipos têm sido relatados como portadores dessa característica (Boiça Jr. \& Lara 1993, Lara et al. 1994)

O trabalho teve por objetivo verificar a resistência de genótipos de sorgo ao ataque de $C$. sorghicola e de $D$. saccharalis e a influência dos mesmos sobre parasitóides.

\section{Material e Métodos}

Os experimentos foram conduzidos na Fazenda de Ensino e Pesquisa da UNESP, campus de Jaboticabal, utilizando-se o delineamento de blocos casualizados com cinco repetições, sendo as parcelas compostas de três linhas de $5 \mathrm{~m}$ de comprimento, espaçadas de $0,6 \mathrm{~m}$.

Realizaram-se dois experimentos com $C$. sorghicola, com o sorgo semeado em 20/10/ 92 ( $1^{\text {a }}$ época) e em 17/12/92 ( $2^{a}$ época), utilizando-se sete genótipos (AF-28, BR-501, BR-601, SC-3541, 3 DX-57, TX-2567 e TX2568). Para a avaliação foram coletadas 12 panículas/parcela, 10 dias após o início do florescimento, quando os grãos estavam leitosos. No laboratório estas panículas foram colocadas em caixas, em número de 6 panículas/caixa. À medida que emergiam, os insetos (C. sorghicola e parasitóides) foram contados e calculada a percentagem de parasitismo.

Realizaram-se dois experimentos adicionais com $D$. saccharalis em semeaduras de 14/12/92 e 12/01/93, utilizando-se os mesmos genótipos, com exceção que no $2^{\circ}$ experimento suprimiu-se o BR-504. Para as avaliações foram coletados 16 colmos, anotando-se o número de internódios, número de internódios broqueados e número de colmos atacados. Calcularam-se a percentagem e a intensidade de infestação. No primeiro experimento foi avaliada também a percentagem de parasitismo por Cotesia flavipes (Cam.); nesse ensaio foram efetuadas duas liberações deste parasitóide, compreendendo aproximadamente 7.500 
indivíduos.

Os números de insetos foram transformados em $(\mathrm{x}+0,5)^{1 / 2} \mathrm{e}$ as percentagens transformadas em arc sen $(\mathrm{P}+0,5)^{1 / 2} \mathrm{e}$ submetidos à análise de variância pelo teste $\mathrm{F}$, sendo as médias comparadas pelo teste de Tukey $(\mathrm{P} \leq 0,05)$.

\section{Resultados e Discussão}

Pela média das duas épocas de plantio verificou-se que o número de $C$. sorghicola foi significativamente mais elevado no genótipo TX-2567 (Tabela 1), evidenciando a suscetibilidade deste material, quando comparados com AF-28, considerada como padrão de alta resistência (Rossetto \& Banzatto 1967, Lara et al. 1977a); 3DX-57, SC-3541 e BR-501 não diferiram de AF-28, sugerindo serem também resistentes; convém ressaltar, no entanto, que o nível de infestação observado foi baixo, nas duas épocas.

Quanto ao número de Tetrastichus sp. nota-se pela média das duas épocas, que o maior índice populacional foi observado em TX-2567, diferindo significativamente dos demais materiais, exceção apenas ao BR-501 com valores intermediários (Tabela 1).

Comparando-se os dados do número de mosca e de parasitóides, das duas épocas, constata-se uma correspondência entre os valores mais elevados no genótipo TX-2567 e menores em AF-28, evidenciando maior presença do parasitóide onde havia maior número de mosca e vice-versa. Quanto à percentagem de parasitismo (Fig. 1), nota-se que foi maior nos genótipos BR-501 $(36,7 \%)$ e AF-28 (20,0\%), na $2^{\mathrm{a}}$ época, sugerindo melhor eficiência do parasitóide nestes materiais que se encontram entre os menos

Tabela 1. Média ( \pm EP) do número Contarinia sorghicola e Tetrastichus sp. em diferentes genótipos de sorgo.

\begin{tabular}{|c|c|c|c|c|c|c|}
\hline \multirow[t]{3}{*}{ Genótipos } & \multicolumn{3}{|c|}{ C. sorghicola ${ }^{1}$} & \multicolumn{3}{|c|}{ Tetrastichus sp. ${ }^{1}$} \\
\hline & \multicolumn{2}{|c|}{ Épocas de plantio } & \multirow{2}{*}{ Média } & \multicolumn{2}{|c|}{ Épocas de plantio } & \multirow{2}{*}{ Média } \\
\hline & Primeira & Segunda & & Primeira & Segunda & \\
\hline TX-2567 & $\begin{array}{c}4,13 \mathrm{a} \\
( \pm 0,46)\end{array}$ & $\begin{array}{c}3,22 \mathrm{a} \\
( \pm 0,36)\end{array}$ & $\begin{array}{c}3,67 \mathrm{a} \\
( \pm 0,36)\end{array}$ & $\begin{array}{c}1,55 \mathrm{a} \\
( \pm 0,27)\end{array}$ & $\begin{array}{c}1,16 a b \\
( \pm 0,19)\end{array}$ & $\begin{array}{c}1,35 \mathrm{a} \\
( \pm 0,16)\end{array}$ \\
\hline TX-2568 & $\begin{array}{l}2,84 \mathrm{ab} \\
( \pm 0,31)\end{array}$ & $\begin{array}{l}2,24 \mathrm{ab} \\
( \pm 0,55)\end{array}$ & $\begin{array}{l}2,54 \mathrm{ab} \\
( \pm 0,50)\end{array}$ & $\begin{array}{c}0,71 \mathrm{~b} \\
( \pm 0,00)\end{array}$ & $\begin{array}{c}0,71 \mathrm{~b} \\
( \pm 0,00)\end{array}$ & $\begin{array}{c}0,71 \mathrm{~b} \\
( \pm 0,00)\end{array}$ \\
\hline BR-601 & $\begin{array}{l}2,45 \mathrm{abc} \\
( \pm 0,30)\end{array}$ & $\begin{array}{l}2,43 \mathrm{ab} \\
( \pm 0,52)\end{array}$ & $\begin{array}{l}2,44 \mathrm{ab} \\
( \pm 0,15)\end{array}$ & $\begin{array}{c}0,71 \mathrm{~b} \\
( \pm 0,00)\end{array}$ & $\begin{array}{l}1,04 \mathrm{ab} \\
( \pm 0,26)\end{array}$ & $\begin{array}{c}0,87 \mathrm{~b} \\
( \pm 0,16)\end{array}$ \\
\hline SC-3541 & $\begin{array}{l}2,37 \mathrm{abc} \\
( \pm 0,35)\end{array}$ & $\begin{array}{c}0,98 \mathrm{~b} \\
( \pm 0,17)\end{array}$ & $\begin{array}{l}1,68 \mathrm{bc} \\
( \pm 0,24)\end{array}$ & $\begin{array}{c}0,91 \mathrm{ab} \\
( \pm 0,10)\end{array}$ & $\begin{array}{c}0,71 \mathrm{~b} \\
( \pm 0,00)\end{array}$ & $\begin{array}{c}0,81 \mathrm{~b} \\
( \pm 0,06)\end{array}$ \\
\hline BR-501 & $\begin{array}{l}1,91 \mathrm{bc} \\
( \pm 0,45)\end{array}$ & $\begin{array}{c}1,66 a b \\
( \pm 0,29)\end{array}$ & $\begin{array}{l}1,78 \mathrm{bc} \\
( \pm 0,40)\end{array}$ & $\begin{array}{c}0,71 \mathrm{~b} \\
( \pm 0,00)\end{array}$ & $\begin{array}{c}1,41 \mathrm{a} \\
( \pm 0,35)\end{array}$ & $\begin{array}{c}1,06 a b \\
( \pm 0,22)\end{array}$ \\
\hline $3 \mathrm{DX}-57$ & $\begin{array}{c}0,71 \mathrm{c} \\
( \pm 0,00)\end{array}$ & $\begin{array}{l}2,60 \mathrm{ab} \\
( \pm 0,48)\end{array}$ & $\begin{array}{l}1,65 \mathrm{bc} \\
( \pm 0,32)\end{array}$ & $\begin{array}{c}0,71 \mathrm{~b} \\
( \pm 0,00)\end{array}$ & $\begin{array}{c}0,81 \mathrm{ab} \\
( \pm 0,10)\end{array}$ & $\begin{array}{c}0,76 \mathrm{~b} \\
( \pm 0,06)\end{array}$ \\
\hline AF-28 & $\begin{array}{c}0,71 \mathrm{c} \\
( \pm 0,00)\end{array}$ & $\begin{array}{c}0,81 \mathrm{~b} \\
( \pm 0,10)\end{array}$ & $\begin{array}{c}0,76 \mathrm{c} \\
( \pm 0,06)\end{array}$ & $\begin{array}{c}0,71 \mathrm{~b} \\
( \pm 0,00)\end{array}$ & $\begin{array}{c}0,81 \mathrm{ab} \\
( \pm 0,10)\end{array}$ & $\begin{array}{c}0,76 \mathrm{~b} \\
( \pm 0,06)\end{array}$ \\
\hline$\overline{\mathrm{F}(\text { tratamento })}$ & $7,00 *$ & $3,73 *$ & $8,11 *$ & $4,08 *$ & $2,92 *$ & $4,37 *$ \\
\hline
\end{tabular}

${ }^{1}$ Médias seguidas de mesma letra não diferem entre si pelo teste de Tukey $(\mathrm{P}<0,05)$; número de insetos/6 panículas. 


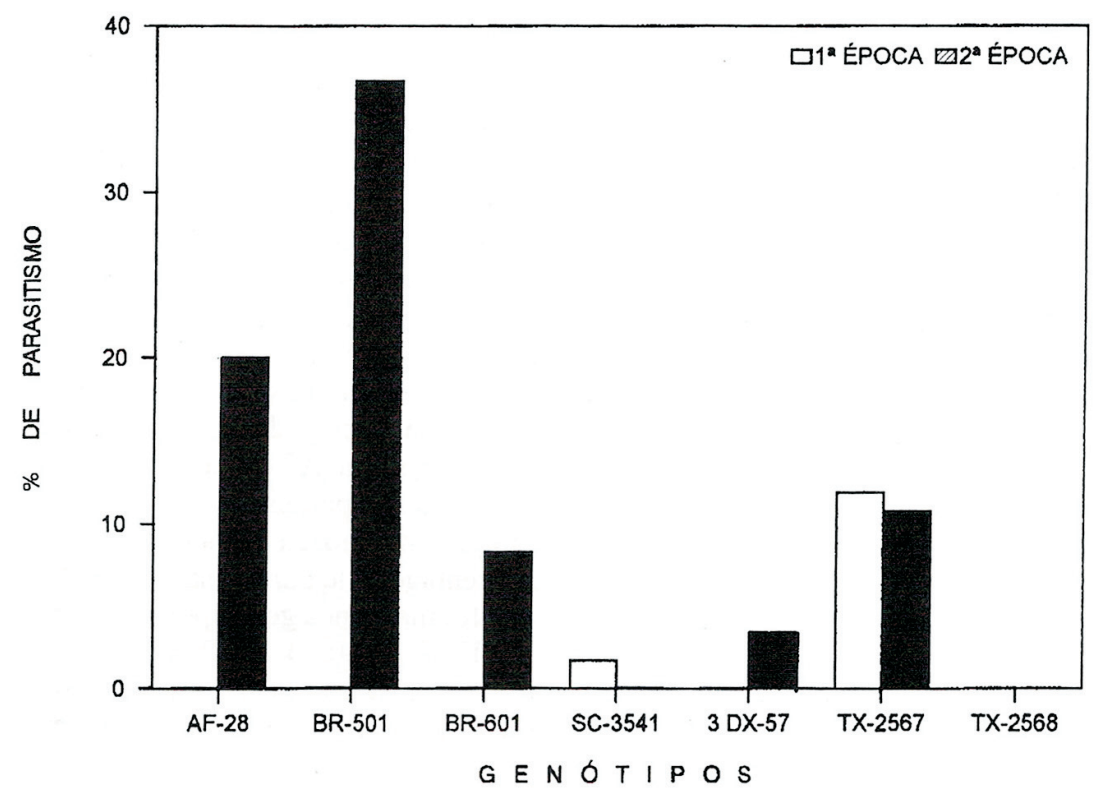

Figura 1. Percentagem de parasitismo de Contarinia sorghicola por Tetrasthicus sp. em diversos genótipos de sorgo, em duas épocas de plantio.

atacados pela mosca, evidenciando bom nível integrado desta praga. Alguns autores já de resistência extrínseca, associação esta haviam constatado maiores níveis de benéfica para futuros programas em manejo parasitismo de C. sorghicola na variedade AF-

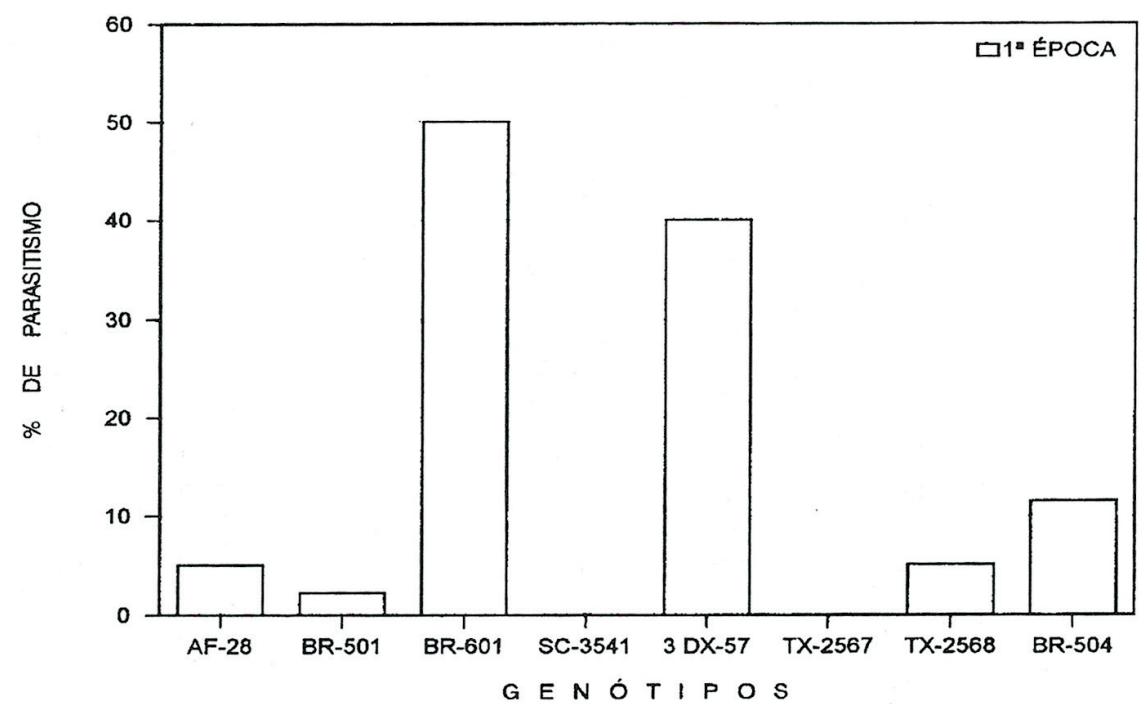

Figura 2. Percentagem de parasitismo de Diatraea saccharalis por Cotesia flavipes em diversos genótipos de sorgo. 
28, como Lara (1974) que observou parasitismo de até $17,6 \%$ nessa variedade, enquanto no híbrido R-1090 o máximo foi de 8,7\%; e como Campos et al (não publicado) que verificaram níveis mais elevados nesse genótipo e no Sart, em relação aos demais, o que confirma a sua resistência extrínseca.

O parasitismo da mosca nos genótipos SC3541 e 3 DX-57 foi baixo e no TX-2568 nulo.
2567. Esses resultados sugerem que os genótipos SC-3541 e AF-28 são resistentes à broca. Com respeito a AF-28, os dados confirmam resultados obtidos por outros autores como Lara et al. (1979), Boiça Jr. \& Lara (1983) e Lara \& Perussi (1984).

Analisando-se a intensidade de infestação, verifica-se que os genótipos $\mathrm{SC}-3541\left(1^{\mathrm{a}}\right.$ época) e AF-28 ( $2^{\mathrm{a}}$ época) foram os que

Tabela 2. Média ( \pm EP) da percentagem de infestação de diferentes genótipos de sorgo por Diatraea saccharalis.

\begin{tabular}{|c|c|c|c|c|}
\hline \multirow[t]{2}{*}{ Genótipos } & \multicolumn{2}{|c|}{ Percentagem de Infestação $^{1}$} & \multicolumn{2}{|c|}{ Intensidade de infestação ${ }^{1}$} \\
\hline & $1^{\mathrm{a}}$ Época & $2^{\mathrm{a}}$ Época & $1^{\mathrm{a}}$ Época & $2^{\mathrm{a}}$ Época \\
\hline TX-2568 & $\begin{array}{c}40,0 \mathrm{a} \\
( \pm 6,43)\end{array}$ & $\begin{array}{l}22,5 \mathrm{ab} \\
( \pm 3,42)\end{array}$ & $\begin{array}{c}4,0 \mathrm{ab} \\
( \pm 0,94)\end{array}$ & $\begin{array}{c}3,0 \mathrm{bc} \\
( \pm 1,24)\end{array}$ \\
\hline BR-504 & $\begin{array}{c}38,8 \mathrm{a} \\
( \pm 6,04)\end{array}$ & - & $\begin{array}{c}5,3 \mathrm{ab} \\
( \pm 0,86)\end{array}$ & - \\
\hline TX-2567 & $\begin{array}{l}25,0 \mathrm{ab} \\
( \pm 2,79)\end{array}$ & $\begin{array}{c}51,3 \mathrm{a} \\
( \pm 8,70)\end{array}$ & $\begin{array}{c}6,1 \mathrm{a} \\
( \pm 1,36)\end{array}$ & $\begin{array}{c}12,6 \mathrm{a} \\
( \pm 0,18)\end{array}$ \\
\hline BR-501 & $\begin{array}{l}25,0 \mathrm{ab} \\
( \pm 7,12)\end{array}$ & $\begin{array}{l}50,0 \mathrm{ab} \\
( \pm 5,59)\end{array}$ & $\begin{array}{c}3,1 \mathrm{ab} \\
( \pm 1,32)\end{array}$ & $\begin{array}{c}7,8 \mathrm{ab} \\
( \pm 1,49)\end{array}$ \\
\hline $3 \mathrm{DX}-57$ & $\begin{array}{l}23,8 \mathrm{ab} \\
( \pm 4,99)\end{array}$ & $\begin{array}{l}35,0 \mathrm{ab} \\
( \pm 9,39)\end{array}$ & $\begin{array}{c}2,4 \mathrm{ab} \\
( \pm 0,65)\end{array}$ & $\begin{array}{c}3,9 \text { bc } \\
( \pm 0,25)\end{array}$ \\
\hline BR-601 & $\begin{array}{l}22,5 \mathrm{ab} \\
( \pm 1,53)\end{array}$ & $\begin{array}{c}28,8 \mathrm{ab} \\
( \pm 10,00)\end{array}$ & $\begin{array}{c}3,0 \mathrm{ab} \\
( \pm 1,89)\end{array}$ & $\begin{array}{c}3,9 \mathrm{bc} \\
( \pm 1,64)\end{array}$ \\
\hline AF-28 & $\begin{array}{l}17,5 \mathrm{ab} \\
( \pm 3,64)\end{array}$ & $\begin{array}{c}18,8 \mathrm{~b} \\
( \pm 3,42)\end{array}$ & $\begin{array}{c}2,2 \mathrm{ab} \\
( \pm 0,64)\end{array}$ & $\begin{array}{c}2,0 \mathrm{c} \\
( \pm 0,47)\end{array}$ \\
\hline SC-3541 & $\begin{array}{c}10,0 \mathrm{~b} \\
( \pm 4,24)\end{array}$ & $\begin{array}{l}32,5 \mathrm{ab} \\
( \pm 5,37)\end{array}$ & $\begin{array}{c}2,0 \mathrm{~b} \\
( \pm 1,02)\end{array}$ & $\begin{array}{l}6,3 \text { abc } \\
( \pm 1,14)\end{array}$ \\
\hline $\mathrm{F}$ (tratamento) & $4,81 *$ & $3,20 *$ & $2,95 *$ & $8,12 *$ \\
\hline
\end{tabular}

${ }^{1}$ Médias seguidas de mesma letra não diferem entre si pelo teste de Tukey $(\mathrm{P} \leq 0,05)$.

A percentagem de parasitismo foi superior na $2^{\mathrm{a}}$ época de plantio em comparação com a $1^{\mathrm{a}}$.

Os dados da percentagem de infestação de $D$. saccharalis (Tabela 2 ), referentes a $1^{\text {a }}$ época de plantio, mostram que dentre os genótipos menos atacados, o SC-3541 foi o único que diferiu estatisticamente de TX-2568 e BR-504 que apresentaram altos índices de infestação; na $2^{a}$ época ocorreu fato semelhante com AF-28 em relação a TX- diferiram significativamente de TX-2567, sugerindo ser este um dos genótipos mais suscetíveis ao ataque de $D$. saccharalis. Quanto a suscetibilidade das variedades BR504 ( $1^{\text {a }}$ época) e BR-501 ( $2^{a}$ época), ela já havia sido observada por Lara \& Perussi (1984) e Pereira et al. (1987), respectivamente.

Quanto à percentagem de parasitismo por C. flavipes (Fig. 2), notam-se índices mais 
elevados nos genótipos BR-601 e 3 DX-57, menores em BR-504, TX-2568, AF-28 e BR501, e nulos em SC-3541 e TX-2567, podendo-se concluir, portanto, que aqueles dois primeiros genótipos mencionados apresentam resistência extrínseca à broca da cana-de-açúcar, considerando-se o parasitóide em questão. A variedade AF-28, que apresenta resistência múltipla, só apresentou resistência extrínseca à mosca do sorgo (i.e., influência positiva da planta sobre o inimigo natural, que a favorece em detrimento da praga).

\section{Agradecimentos}

Os autores expressam seus agradecimentos ao CNPq pelo auxílio, bolsas de pesquisa e bolsa de iniciação científica.

\section{Literatura Citada}

Boiça Jr., A.L. \& F.M. Lara. 1983. Avaliação da resistência de genótipos de Sorghum bicolor (L.) Moench a Diatraea saccharalis (Fabricius, 1794) (Lepidoptera-Pyralidae) e determinação dos tipos envolvidos. An. Soc. Entomol. Brasil 12: 261-272.

Boiça Jr., A.L. \& F.M. Lara. 1993. Resistência de genótipos de sorgo ao ataque de Diatraea saccharalis (Fabr.) (Lepidoptera: Pyralidae). An. Soc. Entomol. Brasil 22: 245-252.

Lara, F.M. 1975. Influência de genótipos de Sorghum vulgare Pers., local e época de plantio, inimigos naturais e inseticidas sobre Contarinia sorghicola (Coquillett, 1898) (Diptera - Cecidomyiidae). Tese de doutorado, FC/UNESP, Baurú, 111p.

Lara, F.M. 1991. Princípios de resistência de plantas a insetos. São Paulo, Ícone, 2 ed., 336 .

Lara, F.M. \& A.L Boiça Jr. 1993. Resistência de genótipos de sorgo ao ataque de Contarinia sorghicola (Coq.,
1898)(Diptera: Cecidomyiidae). Ecossistema 18: 91-100.

Lara, F.M. \& E.M. Perussi. 1984. Resistência de sorgo sacarino Sorghum bicolor (L.) Moench ao ataque de Diatraea saccharalis (Fabr., 1794). Ciênc. Cult. 36: 280-286.

Lara, F.M., A.R. Campos \& E. Berti $F^{0}$. 1994. Preferência de Aprostocetus diplosidis Crawford (Hymenoptera: Eulophidae) por partes da planta de sorgo e por diferentes genótipos. An. Soc. Entomol. Brasil 23: 57-62.

Lara, F.M., C.J. Rossetto \& T. Igue. 1977a. Resistance of the AF-28 sorghum variety to sorghum midge. Entomol. Exp. Appl. 21: $238-242$.

Lara, F.M., G.C. Barbosa F $^{\circ}$ \& J.C. Barbosa. 1980. Danos acarretados por Diatraea saccharalis (Fabr., 1794) na produção de sorgo granífero. Científica 8: 105-111.

Lara, F.M., G.C. Barbosa F ${ }^{\text {o }}$, A.C. Busoli \& J.C. Barbosa. 1979. Comportamento de genótipos de sorgo em relação ao ataque de Diatraea saccharalis (Fabr., 1794). An. Soc. Entomol. Brasil 8: 125130.

Lara, F.M., A.C. Busoli, G.C. Barbosa F ${ }^{\text {, }}$ J. Ayala-Osuna \& D. Perecin. 1977b. Preferência de Diatraea saccharalis (Fabr., 1794) a genótipos de sorgo Sorghum bicolor (L.) Moench, em condições de campo. An. Soc. Entomol. Brasil 6: 58-63.

Pereira, R.P., R.A. da Costa \& J.M. Ferreira. 1987. Incidência de Diatraea em genótipos de sorgo sacarino em diferentes épocas de plantio. Pesq. Agropec. Brasil. 22: 23-25.

Rossetto, C.J. \& N.V. Banzatto. 1967. 
Resistência de sorgo a Contarinia sorghicola (Coquillet) (Diptera : Cecidomyiidae). In Reunião Latinoamericana de Fitotecnia,7, Maracay, Venezuela. p. 292-293.
1967. Praga: fator limitante na produção de sorgo granífero. O Agronômico 19: 12 .

Rossetto, C.J., O. Nakano \& N.V. Banzatto. Recebido em 08/01/96. Aceito em 15/07/97. 